\title{
Bisphosphonate-Related Osteonecrosis in a Patient with Florid Cemento-Osseous Dysplasia
}

\author{
Dong-Jun Seo, Seong-Yong Moon, Jae-Seek You, Ji-Su Oh \\ Department of Oral and Maxillofacial Surgery, College of Dentistry, Chosun University, Gwangju, Korea
}

Received March 17, 2021

Revised March 31, 2021

Accepted April 1, 2021

Correspondence to:

Ji-Su Oh

Department of Oral and Maxillofacial

Surgery, College of Dentistry, Chosun

University, 303 Pilmun-daero, Dong-gu,

Gwangju 61452, Korea

Tel: +82-62-220-3813

Fax: +82-62-222-3810

E-mail: jsoh@chosun.ac.kr

https://orcid.org/0000-0002-8369-5025
Florid cemento-osseous dysplasia (FCOD) is benign, non-neoplastic lesion characterized by multiple sclerosing masses. Cases of bisphosphonate-related osteonecrosis in FCOD have been rarely reported. we present the patient had multiple osteonecrosis with FCOD in the jaw that occurred after receiving bisphosphonates for eight years due to osteoporosis This report discussed the importance of evaluation of the bone disease in the jaw before bisphosphonate treatment and periodic follow-up.

Key Words: Bisphosphonate-associated osteonecrosis of the jaw; Jaw; Osteomyelitis; Osteoporosis

\section{INTRODUCTION}

Benign fibro-osseous lesions refer to a group of states characterized by replacing the normal bone with a fibrous connective tissue that shares the same basic evolutive mechanism and becomes gradually mineralized [1]. Among them, cemento-osseous dysplasia (COD) of the jaws originates as a periodontal ligament element and is a fairly common lesion [2], and COD is still a controversial term [3]. According to the latest (4th) edition of the World Health Organization (WHO) classification of head and neck tumors (2017) [3], WHO renames back to the terminology COD, to accept them as odontogenic with an origin in the periodontal ligament. There are three categories: periapical COD, focal COD, and florid COD (FCOD) [3].

FCOD is characterized by multiple sclerosing masses only in jaws, which is described as a benign, non-neoplastic lesion [2]. The word 'florid' was introduced to describe the extensive, widespread manifestations in the jaws [4]. The classical FCOD has histologically dense, highly mineralized characteristics and appears almost acellular cemento-osseous tissue [5]. Clinically, these multiple lesions are often asymptomatic and can appear as incidental radiological findings [6]. So the disease may exist for many years without symptoms [6]. Radiologically, lesions have radiolucent margins with varying degrees of opacity around sclerotic mass $[5,6]$. The disease has self-limiting characteristics, affects only the alveolar processes, and is independent of teeth [6]. FCOD tends to occur predominantly in middle-aged black women [6]. The disease is prone to secondary infections when exposed to oral pathogens due to the dense sclerotic hypovascular characteristic, which is difficult to manage [7]. So extensive surgical debridement may be required [7].

There are two treatments to manage FCOD. It is recommended that asymptomatic patients be observed without surgical intervention. Symptoms usually occur by exposing a sclerotic mass to the oral cavity, so biopsy or surgical extraction of teeth in the affected area should be avoided. No invasive treatment should be performed in asymptomatic lesions. If there are no clinical signs, reevaluation with 
panoramic imaging is adequate once every 2 or 3 years. If new signs or symptoms are observed, examination with dental computed tomography (CT) imaging should be considered. Chronic inflammation and infection appear within dense mineralized tissue, making it more difficult to manage the symptomatic patients. These infections are known to be difficult to eradicate and often require extensive surgical debridement and saucerization. Although antibiotic therapy can be helpful in FCOD, it is often ineffective because the involved area is composed of dense mineralized tissue that is not well perfused [7-9].

Cases of infected FCOD have been rarely reported, especially bisphosphonate-related osteonecrosis in FCOD. In this rare case, we reported the patient with FCOD who presented multiple osteonecrosis that occurred after receiving bisphosphonates for eight years due to osteoporosis. This study was conducted in accordance with the principles of the Declaration of Helsinki, and patient provided written informed consent.

\section{CASE REPORT}

A 60-year-old female patient was referred with a chief complaint of pain and pus discharge in the left posterior mandible over the past two months. She had diabetes mellitus, anemia, hypothyroidism, and osteoporosis. The patient was diagnosed asymptomatic FCOD with multiple diffuse densely sclerotic lesions confused with roots based on the clinical and radiographic findings before six years (Fig. 1). Further treatments were not planned, periodic followup was lost for six years. When the patient returned to the hospital six years later, the patient presented that she had been taking risedronate monthly for eight years to treat osteoporosis.

Intraoral examination revealed severe gingival swelling, exposure of alveolar bone, and yellow, thick purulent discharge.

In cone-beam CT and panoramic radiographs, a wide range of lobulated dense mixed radiopaque-radiolucent lesions were observed throughout the right maxilla and left mandible (Fig. 2). The lesions included the right first and second molars in the maxilla (Fig. 3A), and continued from the left second premolar to the third molar in the mandible (Fig. 3B). The sclerotic lesions which were not separated and connected like cotton-wool were surrounded by wide osteolytic radiolucent lesions. The roots involved in the lesions were confused without resorption. The destruction of the alveolar bone without expansion was extended just above the inferior alveolar canal, but there were fortunately not interruptions of the canal. The alkaline phosphatase level (50 units/L) and white blood cell count $\left(5.14 \times 10^{3} / \mu \mathrm{L}\right)$ was

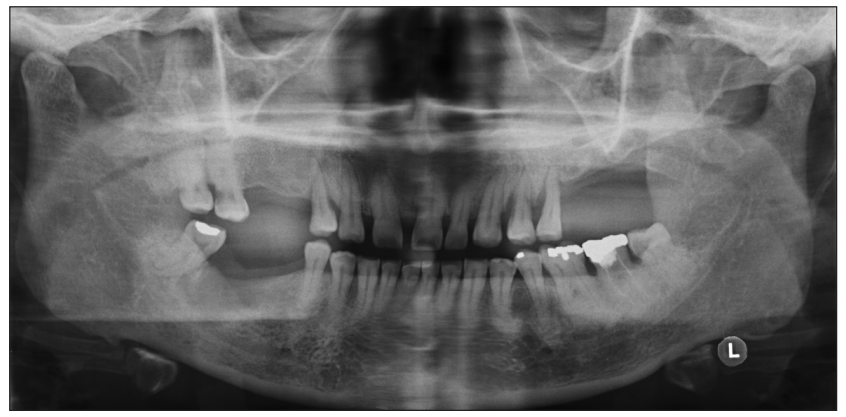

Fig. 1. Panoramic radiograph showing multiple diffuse densely sclerotic lesions confused with roots before six years.

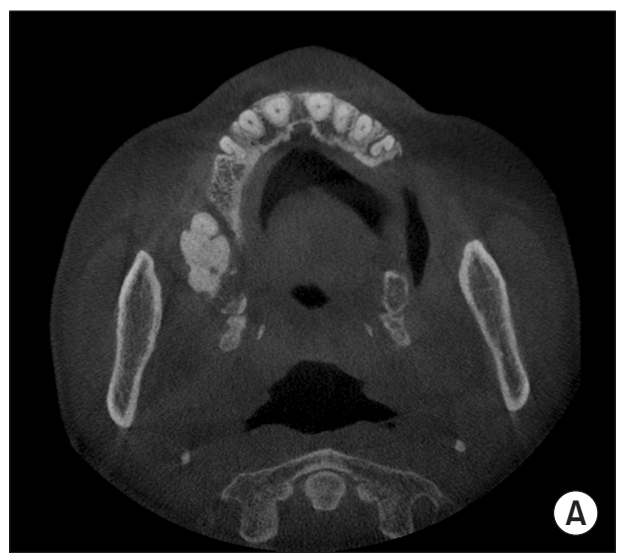

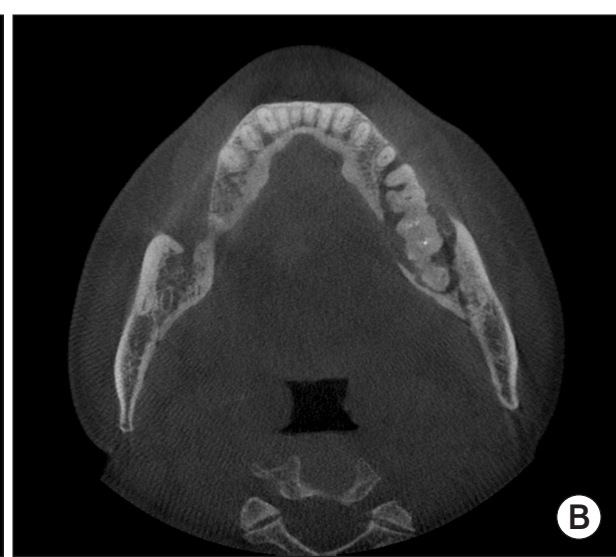

Fig. 2. Axial view computed tomography scan demonstrating a wide range of dense mixed radiopaque-radiolucent lesions without bony expansion in the right maxilla (A) and left mandible (B). 


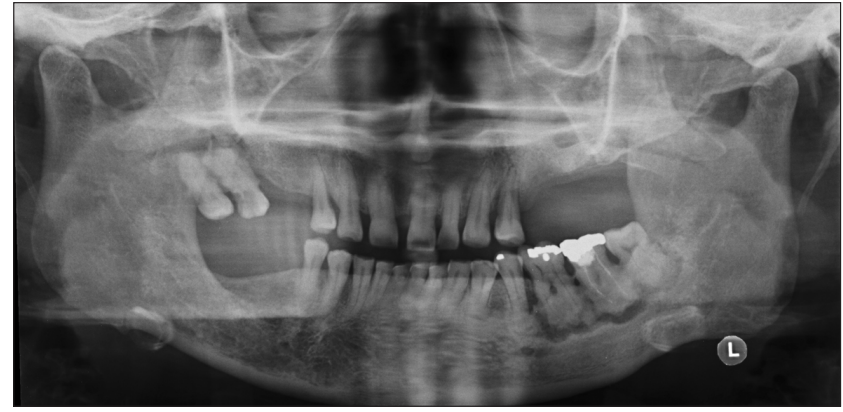

Fig. 3. Panoramic radiograph revealed sclerotic lesions confused with root not separated and connected to each other surrounded by wide osteolytic radiolucent lesions.

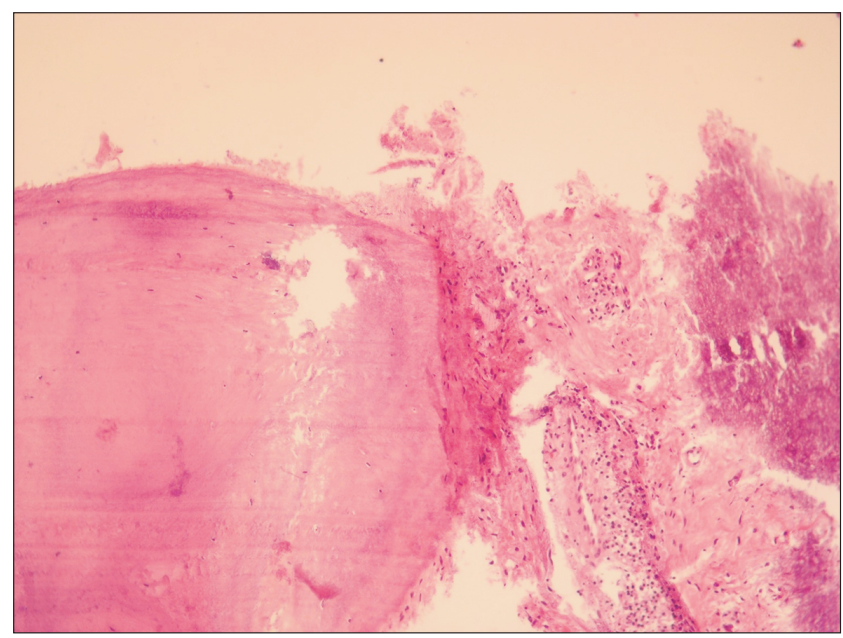

Fig. 4. Histopathologic feature showing cementum-like calcification structure around inflammatory cell infiltration (H\&E staining, $\times 200$ ).

revealed normal range. Thyroid stimulating hormone was low $(0.10 \mu \mathrm{U} / \mathrm{mL})$ and result of free $\mathrm{T} 4(1.38 \mathrm{ng} / \mathrm{dL})$ and $\mathrm{T} 3$ $(94.7 \mathrm{ng} / \mathrm{dL})$ were normal.

The tentative diagnosis of medication-related osteonecrosis of the jaw (MRONJ) complicating FCOD was given. Based on tentative diagnosis, the drug holiday of bisphosphonate, the prescription of anti-inflammatory and antibiotic medication, and the instruction of oral hygiene were planned. The gingival swelling and purulent discharge were subsided after three months. Surgical removal of necrotic bone and curettage was performed with the extraction of the involved teeth under general anesthesia.

On histopathological examination, fibrous connective tissue, neutrophils, and granulation tissue are observed around the spherical cementum-like calcification structure (Fig. 4). Combined with clinical and radiological findings, both the

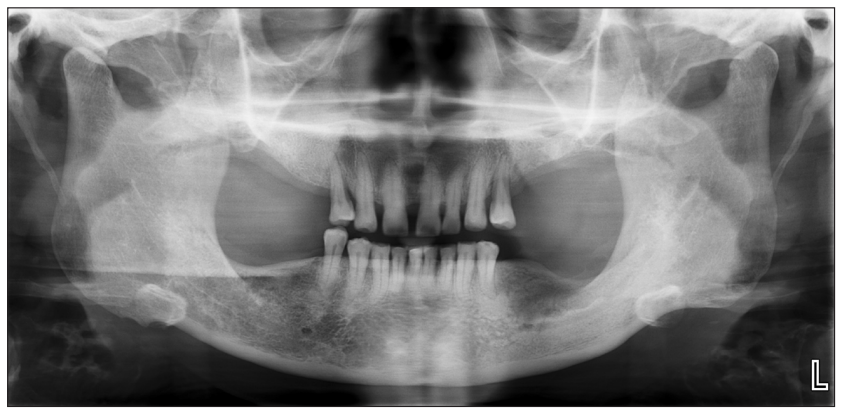

Fig. 5. Panoramic radiograph showing a normal bone structure in the right maxilla and left mandible after 1 year of follow-up.

left mandibular and right maxillary lesions were confirmed as MRONJ associated with infected FCOD. Complete healing was observed 12 months after surgery, and there was no evidence of bone exposure or infection (Fig. 5).

\section{DISCUSSION}

Bisphosphonates are administered to malignant neoplasms, such as multiple myeloma and osteolytic diseases, such as osteoporosis, osteoarthritis. Bisphosphonates suppress bone resorption by reducing the activity of osteoclasts, increasing the apoptosis of osteoclasts, and decreasing the regulation of matrix metalloproteinases. However, as a result of local sclerosis and inhibition of bone remodeling, patients with severe osteomyelitis of the jaw resulted from its use have been reported [10-13]. The first cases of bisphosphonate-related osteonecrosis of the jaws (BRONJ) were reported in 2003 by Marx [13] and 2004 by Ruggiero et al. [11] Since then, other anticancer (i.e., sirolimus, bevacizumab) and antiresorptive (denosumab) medications have been implicated in jaw necrosis; as of 2014, Special Committee of the American Association of Oral and Maxillofacial Surgeons (AAOMS) has named this diagnosis as MRONJ. MRONJ can be defined as 1) current or previous treatment with antiangiogenic and/or antiresorptive agents; 2) no history of radiation therapy or no eminent metastatic disease to the jaw; and 3) exposed bone probed through an intraoral or extraoral fistula continuing for more than eight weeks [14].

BRONJ accounts for the largest percentage of MRONJ. The precise mechanism of BRONJ is not yet unknown. It seems to be related to the defects in the physiological 
remodeling or healing of the jaw. Especially, because of the anti-angiogenic properties of bisphosphonates, problems with blood supply to the bone and ischemic changes can occur locally in the affected jaw $[11,15]$.

Dentists should be aware of the key signs and symptoms: pain, halitosis, exposed necrotic bone, hypoesthesia or paresthesia, mobility of teeth, and signs of infection such as cellulitis, swelling, fistula, and pus exudation [11,15]. And also, dentists need to know the risk factors that can exacerbate the development and severity of MRONJ. The primary risk factor for MRONJ is exposure to denosumab or bisphosphonates. Local infection or trauma to the bone or soft tissue (usually surgical trauma or pressure sores) develops the condition of MRONJ. In many studies, systemic chronic diseases, the situation of immunocompromise (ie, acquired immune deficiency syndrome), and diseases that reduce the vascularization of the bone seem to affect osteomyelitis. Smoking, the use of corticosteroids, alcohol abuse, intravenous drugs, radiation, poor oral hygiene, diabetes mellitus, exanthematous fever, anemia, and malignant diseases have been considered to be related to increased frequency of osteomyelitis [10,11,13,14,16].

When it comes to managing symptoms, MRONJ can be managed effectively, and if precautions are taken, the risk of worsening the condition can be greatly reduced. It is advisable for patients, dentists, and physicians to consider prophylactic measures to reduce the risk of MRONJ. If the patients with bone metastases are going to need treatment with denosumab or bisphosphonate, they should fully inform about the risk of developing MRONJ by physicians. Also, before the administration of denosumab or bisphosphonates, the patients should also undergo a clinical and radiological assessment of overall oral hygiene by their dentist. An important key strategy is to keep the patient's overall oral hygiene good and to have the patient undergo the dental evaluation by dentist every 6 months [11,13-16].

In the event of dental infection such as caries, periodontitis despite these precautions, timely diagnosis and resolution are important in preventing the deterioration of the condition. Extractions should be conducted with the lowest possible trauma level and be performed in a surgical setting to reduce the risk of exacerbating MRONJ. And treatments should be carried out under prophylactic antibiotics and performed by smoothening of sharp bone and clear closure of the wounds, and then observed until complete soft tissue healing. In stage 1, symptoms and signs of MRONJ can be effectively managed in most patients through a conservative approach. According to the current AAOMS guidelines, debridement is recommended to relieve soft tissue irritation and control infection at stage 2, and resection is recommended at stage 3. At later stages, the removal of necrotic bone, sequestrectomy, smoothing of sharp bone margin, and wound closure may be required; some patients may need segmental/continuity resections. Traditionally, surgical treatment is recommended after sequestrum is formed. However, surgery can be effective in the treatment of MRONJ at any stage. Some physicians recommend stopping the administration of bisphosphonates or denosumab for 2 or 3 months for drug holidays for local treatment and healing after surgery. But there is no clear evidence to support the optimal timing or the effectiveness and safety of this method [11-16].

Lesions such as FCOD are vulnerable to secondary infection because they have hypovascularized and dense sclerosis properties when exposed to oral pathogens, and can be difficult to manage. Therefore, patients must undergo continuous evaluation and management. Extensive surgical treatment may be necessary if adequate immediate treatment is not possible after exposure to the infection. In particular, the surgery of the cases of MRONJ combined with FCOD may be aggressive than the cases of MRONJ alone. As in this case, in patients with FCOD who have been taking bisphosphonate for a long time, problems with osteoclasts can degrade the proper healing process for infection and trauma, which can lead to fatal multiple osteomyelitis or osteonecrosis. Therefore, if an early finding of FCOD, the dentist must perform a periodic follow-up and thorough hygiene management in the case of asymptomatic patients, and appropriate treatment should be performed in the case of symptomatic patients. Also, dentists must understand the overall understanding of MRONJ and know how to treat it.

In conclusion, the bone disease in the jaw should be evaluated before bisphosphonate is administered. Also, periodic follow-up and early diagnosis of MRONJ are emphasized in FCOD patients who are given drugs associated with MRONJ. 


\section{CONFLICT OF INTEREST}

No potential conflict of interest relevant to this article was reported.

\section{ORCID}

\author{
Dong-Jun Seo \\ https://orcid.org/0000-0002-4301-5881 \\ Seong-Yong Moon \\ https://orcid.org/0000-0002-7513-4404 \\ Jae-Seek You \\ https://orcid.org/0000-0001-7638-9583 \\ Ji-Su Oh \\ https://orcid.org/0000-0002-8369-5025
}

\section{REFERENCES}

1. de Noronha Santos Netto J, Machado Cerri J, Miranda AM, Pires FR. Benign fibro-osseous lesions: clinicopathologic features from 143 cases diagnosed in an oral diagnosis setting. Oral Surg Oral Med Oral Pathol Oral Radiol 2013;115:e56-e65.

2. Coleman H, Altini M, Kieser J, Nissenbaum M. Familial florid cemento-osseous dysplasia--a case report and review of the literature. J Dent Assoc S Afr 1996;51:766-770.

3. El-Mofty SK, Nelson B, Toyosawa S, Wright JM. Cemento-osseous dysplasia. In: El-Naggar AK, Chan JKC, Grandis JR, Takata T, Slootweg PJ, eds. WHO classification of head and neck tumours. 4th ed. Lyon: IARC Press; 2017. pp. 254-255.

4. Mangala M, Ramesh DN, Surekha PS, Santosh P. Florid cementoosseous dysplasia: review and report of two cases. Indian J Dent Res 2006;17:131-134.

5. Ong ST, Siar CH. Florid cemento-osseous dysplasia in a young Chinese man. Case report. Aust Dent J 1997;42:404-408.
6. Melrose RJ, Abrams AM, Mills BG. Florid osseous dysplasia. A clinical-pathologic study of thirty-four cases. Oral Surg Oral Med Oral Pathol 1976;41:62-82.

7. Fenerty S, Shaw W, Verma R, et al. Florid cemento-osseous dysplasia: review of an uncommon fibro-osseous lesion of the jaw with important clinical implications. Skeletal Radiol 2017;46:581590.

8. Beylouni I, Farge P, Mazoyer JF, Coudert JL. Florid cementoosseous dysplasia: report of a case documented with computed tomography and 3D imaging. Oral Surg Oral Med Oral Pathol Oral Radiol Endod 1998;85:707-711.

9. Waldron CA. Fibro-osseous lesions of the jaws. J Oral Maxillofac Surg 1993;51:828-835.

10. Cavalcante MB, de Oliveira Lima AL, Júnior MA, Santos MB. Florid cemento-osseous dysplasia simultaneous the chronic suppurative osteomyelitis in mandible. J Craniofac Surg 2016;27: 2173-2176.

11. Ruggiero SL, Mehrotra B, Rosenberg TJ, Engroff SL. Osteonecrosis of the jaws associated with the use of bisphosphonates: a review of 63 cases. J Oral Maxillofac Surg 2004;62:527-534.

12. Senel FC, Saracoglu Tekin U, Durmus A, Bagis B. Severe osteomyelitis of the mandible associated with the use of non-nitrogencontaining bisphosphonate (disodium clodronate): report of a case. J Oral Maxillofac Surg 2007;65:562-565.

13. Marx RE. Pamidronate (Aredia) and zoledronate (Zometa) induced avascular necrosis of the jaws: a growing epidemic. J Oral Maxillofac Surg 2003;61:1115-1117.

14. Ruggiero SL, Dodson TB, Fantasia J, et al. American Association of Oral and Maxillofacial Surgeons position paper on medicationrelated osteonecrosis of the jaw--2014 update. J Oral Maxillofac Surg 2014;72:1938-1956.

15. Migliorati CA, Schubert MM, Peterson DE, Seneda LM. Bisphosphonate-associated osteonecrosis of mandibular and maxillary bone: an emerging oral complication of supportive cancer therapy. Cancer 2005;104:83-93.

16. Otto S, Pautke C, Van den Wyngaert T, Niepel D, Schiødt M. Medication-related osteonecrosis of the jaw: prevention, diagnosis and management in patients with cancer and bone metastases. Cancer Treat Rev 2018;69:177-187. 